\title{
Aerodynamic drag determination of a full-scale cyclist mannequin from large-scale PTV measurements
}

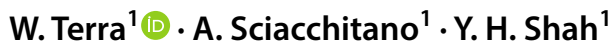

Received: 23 August 2018 / Revised: 7 January 2019 / Accepted: 8 January 2019 / Published online: 21 January 2019

(c) The Author(s) 2019

\begin{abstract}
The aerodynamic drag of a human-scale wind tunnel model is obtained from large-scale particle tracking velocimetry measurements invoking the conservation of momentum in a control volume surrounding the model. Lagrangian particle tracking is employed to obtain the velocity and static pressure statistics in a thin volume in the wake of a cyclist mannequin at freestream velocities between 12.5 and $15 \mathrm{~m} / \mathrm{s}$, corresponding to Reynolds numbers from $5 \times 10^{5}$ to $6 \times 10^{5}$ based on the torso length. The spatial distributions of the time-average streamwise velocity and pressure coefficient match well with previous works reported in literature. The streamwise velocity fluctuations in the wake of the cyclist's model are presented, clearly demonstrating the unsteady nature of the main wake flow structures. Furthermore, the obtained aerodynamic drag follows the expected quadratic increase with increasing freestream velocity. The accuracy of this drag estimation is evaluated by comparison to force balance data and corresponds to $30 \mathrm{drag}$ counts. The three terms composing the overall drag force, ascribed to the mean and fluctuating streamwise velocity and the mean pressure, are also evaluated separately, demonstrating that the resistive force is dominated by the contribution of the mean streamwise momentum deficit, whereas the contribution of the pressure term is negligible.
\end{abstract}

\section{Graphical abstract}

\section{Aerodynamic drag determination of a full-scale cyclist mannequin from large-scale PTV measurements}
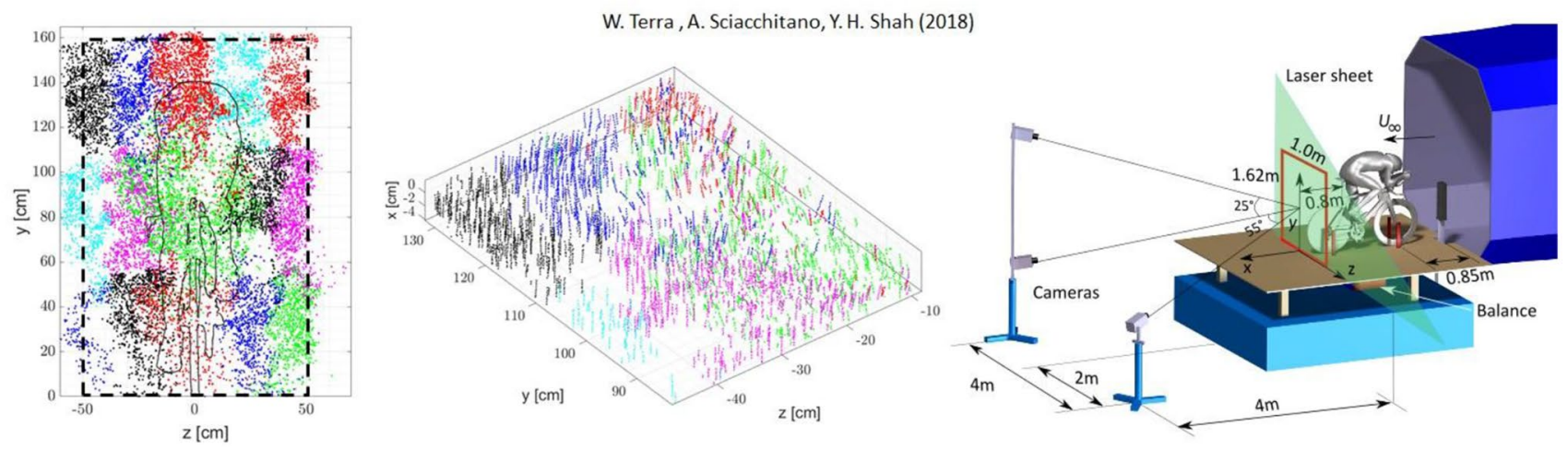

The data presented in the figures in this work is available online (https://doi.org/10.4121/uuid:7f42e060-766d-4bf1-86c4-c648c $794317 \mathrm{c})$

\section{W. Terra}

w.terra@tudelft.nl

1 Aerospace Engineering Department, Delft University of Technology, Delft, The Netherlands

\section{Introduction}

Determination of the aerodynamic loads is relevant in many fluid dynamic applications, e.g., for the fuel-efficient design of air and ground transportation systems, the safe structural design of wind turbines and the maximization of performances in elite speed sports such as cycling and skating. 
Measurement of these aerodynamic loads is conventionally carried out in wind tunnels, where full-scale or scaled-down models are immersed in a homogeneous stream of air, while the forces and moments are measured via six-component force balances (e.g., Zdravkovich 1992; Tropea et al. 2007). Thanks to their high resolution (up to $0.0003 \%$ of the full scale load, Tropea et al. 2007) these balance systems are nowadays considered as standard tools especially for measurements in industrial wind tunnels. Nevertheless, such balance measurements are regarded as "blind", in the sense that they do not provide insight in the flow field and flow structures generating these aerodynamic loads. Alternatively, the aerodynamic loads can be evaluated using wake rakes, invoking the conservation of momentum across a control volume containing the model and measuring the total and static pressure in the wake of the model (Betz 1925; Jones 1936; Goett 1938; Somers 1997, among others). In contrast to balance measurements, the wake rake approach provides not only the aerodynamic forces, but also allows relating these forces to the flow variables in the wake of the model, yielding a deeper insight into the components and generation of the total resistive force (e.g., Maskell 1973; Hucho and Sovran 1993). These pressure-based wake rake measurements, however, are intrusive in nature and yield the result at a single point in space, requiring traversing mechanisms and precluding measurement of the instantaneous flow field.

In the last two decades Particle Image Velocimetry (PIV) has been used extensively as a viable alternative to the pressure probe wake rakes, for loads determination from wake velocity data, primarily due to its whole-field measurement capability and non-intrusive nature. Lin and Rockwell (1996) and Unal et al. (1997) conducted PIV measurements in the wake of a two-dimensional cylinder to characterize its unsteady lift coefficient at $R e=3780$. Similarly, Noca et al. (1997) and Kurtulus et al. (2007) used time-resolved PIV to quantify the unsteady aerodynamic forces of cylinders at $R e=100$ and $R e=4890$, respectively. Using PIV velocity data and the control volume approach, van Oudheusden et al. (2006) characterized the time-average aerodynamic forces and pitching moment of an airfoil; the authors reported a discrepancy between the drag coefficient measured with the PIV wake rake and the conventional pressure-based wake rake of 1 drag count (or $\Delta C_{D}=10^{3}$ ). Ragni et al. (2009) proved the feasibility of the PIV wake rake for drag determination of a transonic airfoil at Mach $=0.6$. In a successive work, the authors extended the use of the PIV wake rake to moving objects for the determination of the aerodynamic loads on an aircraft propeller blade (Ragni et al. 2011). Recently, a detailed review of loads estimation approaches from PIV measurements has been carried out by Rival and van Oudheusden (2017) and a set of guidelines is provided for accurate fluid force measurements involving unsteady body motion by Lentink (2018).

Despite the popularity of the PIV wake rake for measuring the time-average and instantaneous loads and investigation of the governing flow fields, its application has been limited to relatively small sized wind tunnel models (typical characteristic length of the order of $10 \mathrm{~cm}$ ) due to the limited domain of conventional PIV measurements (Raffel et al. 2018). This is largely ascribed to the low scattering efficiency of conventional micrometric flow tracers. The introduction of sub-millimeter Helium-filled soap bubbles (HFSB) as flow tracers for PIV measurements (Bosbach et al. 2009; Scarano et al. 2015) allowed a dramatic increase in measurement size (square meters and liters for planar and volumetric PIV, respectively), making 'large-scale' PIV measurements possible in wind tunnels. Large-scale PIV has mainly been exploited for volumetric PIV measurements. Caridi et al. (2016) made use of largescale tomographic PIV over a volume of 121 to investigate the flow field at the tip of a vertical axis wind turbine blade. By conducting large-scale PTV measurements and solving the Poisson equation for pressure, Schneiders et al. (2016) measured the instantaneous volumetric pressure in the wake of a truncated cylinder over a volume of about 6 liters. Loads determination from large-scale PIV has been attempted for the first time by Terra et al. (2017), estimating the drag coefficient of a transiting sphere at $R e=10,000$. Load determination from large-scale PIV in wind tunnels, however, has been hampered firstly by the limited HFSB tracers concentration, which has been below 1 bubble $/ \mathrm{cm}^{3}$ (Caridi et al. 2016), and secondly by the limited size of the seeded stream tube cross-section, not exceeding the order of $0.1 \mathrm{~m}^{2}$ (Caridi et al. 2016; Jux et al. 2018).

The present work aims to assess the feasibility of using a large-scale PIV wake rake for the determination of the aerodynamic drag on a three-dimensional human-scale wind tunnel model. For this purpose, Lagrangian particle tracking is employed to obtain the velocity in a plane of approximately $1.6 \mathrm{~m}^{2}$ in the wake of a full-scale cyclist mannequin, demonstrating the human-scale PIV wake rake on a fully three-dimensional, unsteady and highly complex flow, recently discussed in several studies (Defraeye et al. 2010; Crouch et al. 2014; Barry et al. 2014, among others). The obtained time-average flow topology is presented and validated against literature. Furthermore, for the first time the distribution of streamwise velocity fluctuations and experimentally measured pressure in the wake plane are presented and discussed. Finally, the accuracy of the aerodynamic drag is evaluated by comparison with state-of-the-art balance measurements and repeating the measurements at varying freestream velocity. 


\section{Theoretical background}

Consider a body in relative motion with respect to a fluid. In the incompressible flow regime, the instantaneous drag of the object can be determined invoking the conservation of momentum in a control volume enclosing the body (Anderson 1991):

$D(t)=-\rho \iiint_{V} \frac{\partial u}{\partial t} \mathrm{~d} V+\rho \iiint_{S_{\text {wake }}}\left(U_{\infty}-u\right) u \mathrm{~d} S+\iint_{S_{\text {wake }}}\left(p_{\infty}-p\right) \mathrm{d} S$,

where $V$ is the control volume, $S_{\text {wake }}$ the downstream boundary of the control volume extending sufficiently far into the freestream, $\rho, p_{\infty}$ and $U_{\infty}$ are the fluid density, freestream pressure and freestream velocity, respectively, and $p$ and $u$ are the static pressure and stream-wise velocity at the location $S_{\text {wake }}$ as illustrated in Fig. 1.

Applying Reynolds decomposition to the velocity and pressure and averaging both sides of Eq. 1, the time-averaged drag is obtained:

$\bar{D}=\rho \iint_{S_{\text {wake }}}\left(U_{\infty}-\bar{u}\right) \bar{u} \mathrm{~d} S-\rho \iint_{S_{\text {wake }}} \overline{u^{\prime 2}} \mathrm{~d} S+\iint_{S_{\text {wake }}}\left(p_{\infty}-\bar{p}\right) \mathrm{d} S$,

where $\bar{u}$ is the time-average streamwise velocity, $u^{\prime}$ the fluctuating streamwise velocity and $\bar{p}$ the time-average static pressure. Following Terra et al. (2017), the first, second and third term at the right hand side of this equation are referred to as the momentum term, the Reynolds stress term and the pressure term, respectively. Equation 2 allows evaluating the time-average aerodynamic drag from velocity and pressure statistics in the wake of the wind tunnel model. The latter is computed from the PIV data by solving the Poisson equation for pressure, which is obtained after Reynolds averaging the momentum equation and prescribing appropriate boundary conditions (van Oudheusden 2013). The viscous term has been omitted in this reconstruction assuming its contribution is negligible (de Kat and van Oudheusden 2012).

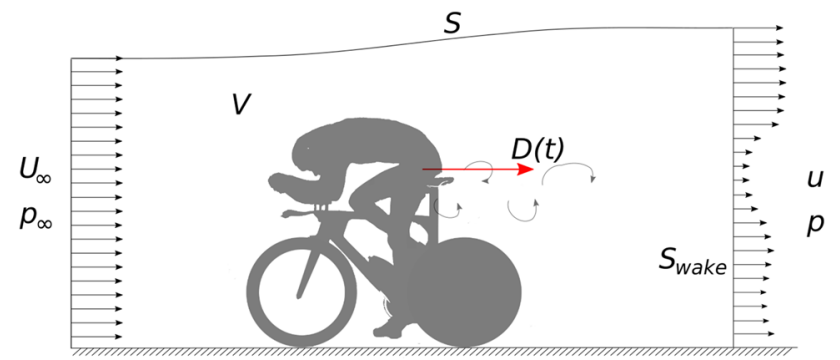

Fig. 1 Schematic of the control volume approach to determine the aerodynamic drag of an object
The accuracy of the drag estimation via the PIV wake rake approach is assessed via direct comparison with stateof-the-art balance measurements. The measurements are repeated at different freestream velocity in a narrow range of Reynolds numbers, where the drag coefficient is assumed to be constant. The drag resolution of the PIV wake rake is evaluated as:

$\Delta C_{D}=\sqrt{\frac{1}{N} \sum_{i=1}^{N}\left(\overline{C_{D_{i, \mathrm{PIV}}}}-\overline{C_{D_{i, \mathrm{bal}}}}\right)^{2}}$,

where $\overline{C_{D_{i, \mathrm{PIV}}}}$ and $\overline{C_{D_{i, \mathrm{bal}}}}$ are the time-average drag coefficients from the PIV wake rake and the balance system, respectively, and $N$ is the number of repeated measurements at different freestream velocities.

\section{Experimental apparatus and procedure}

\subsection{Wind tunnel model}

The experiments are conducted in the Open Jet Facility (OJF) of the Aerodynamics Laboratories at the Delft University of Technology. This atmospheric closed-loop, open jet wind tunnel has an octagonal cross-section of $2.85 \times 2.85 \mathrm{~m}$ with a contraction ratio of $3: 1$, that allows the generation of a homogeneous jet at speeds between 4 and $35 \mathrm{~m} / \mathrm{s}$ with $0.5 \%$ turbulence intensity (Lignarolo et al. 2014). The wind tunnel model consists of a rigid-body full-scale cyclist mannequin seated on a time-trial bike. The latter is supported at the front and rear axis, as illustrated in Fig. 2, with the front wheel centreline located $1 \mathrm{~m}$ downstream of the OJF contraction exit. The mannequin, wearing a long-sleeved Etxeondo time trial suit along with a Giant time trial helmet (season 2016), is manufactured from thermoplastic polyester by additive manufacturing after scanning an elite cyclist in

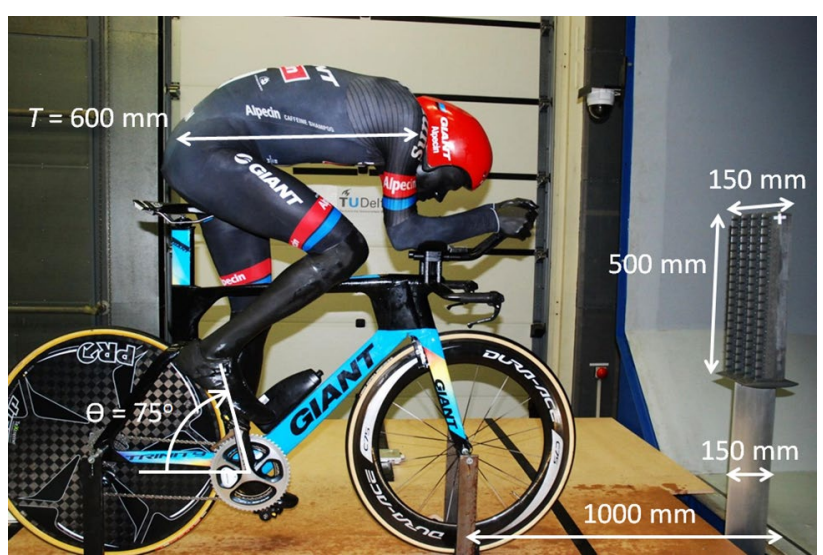

Fig. 2 Wind tunnel model and PIV seeding system 
time-trial position (Van Tubergen et al. 2017). The legs of the mannequin are in asymmetric position (left leg stretched and right leg bended) relating to a $75^{\circ}$ crank angle (Fig. 2). The model's hip width $W$, torso length $T$ and frontal area $A$ are $0.365 \mathrm{~m}, 0.600 \mathrm{~m}$ and $0.32 \mathrm{~m}^{2}$, respectively. More details of the mannequin dimensions are available in the work of Terra et al. (2016) and Jux et al. (2018). A $4.9 \mathrm{~m}$ long and $3.0 \mathrm{~m}$ wide wooden table, elevated $20 \mathrm{~cm}$ above the wind tunnel contraction exit, is used to reduce the boundary layer thickness interacting with the model.

\subsection{PIV system}

The flow field in the wake of the cyclist model is measured by Lagrangian particle tracking with neutrally buoyant helium-filled soap bubbles (HFSB) as flow tracers. The latter have a diameter of approximately $300 \mu \mathrm{m}$ (Scarano et al. 2015) and are introduced into the flow by an in-house developed seeding rake installed on a two-axis traversing system at the exit of the wind tunnel contraction (Fig. 2). 80 HFSB generators are integrated into the four-wing seeding rake with a vertical and horizontal pitch of $25 \mathrm{~mm}$ and $50 \mathrm{~mm}$, respectively. The trailing edge of the rake is installed $85 \mathrm{~cm}$ upstream of the front wheel's axis (see Fig. 2) and releases approximately $2 \times 10^{6}$ bubbles per second, seeding a streamtube of approximately $20 \times 50 \mathrm{~cm}^{2}$ cross-section in the freestream. At a freestream velocity of $14 \mathrm{~m} / \mathrm{s}$, the seeding concentration is estimated at $1.4 \mathrm{tracer} / \mathrm{cm}^{3}$ (Caridi et al. 2016). The flow rates of helium, air and bubble fluid solution are regulated via a Fluid Supply Unit from LaVision $\mathrm{GmbH}$. To seed the entire wake of the model, measurements are repeated at 15 different positions of the seeding rake, 5 along the horizontal direction and 3 along the vertical direction. Due to the presence of the seeding rake, the freestream turbulence intensity, measured $2 \mathrm{~m}$ downstream of the seeding rake, is increased from 0.5 to $1.9 \%$, while the mean flow remains unaltered (Jux et al. 2018). Details on the different seeder positions and the effect of the seeding rake on the measured aerodynamic drag are provided in the "Appendix".

Collimated light is provided by a Continuum Mesa PIV 532-120-M laser (Nd:YAG diode pumped, pulse energy of $18 \mathrm{~mJ}$ at $1 \mathrm{kHz}$ ) illuminating a $5 \mathrm{~cm}$ thick plane, $80 \mathrm{~cm}$ downstream of the trailing edge of the saddle of the bike (see Fig. 3). Time-resolved images are acquired by three Photron FastCAM SA1 cameras (CMOS sensor, 12 bit, $20 \mu \mathrm{m}$ pixel pitch, $1024 \times 1024$ pixels at full resolution) over a region of $1.0 \times 1.62 \mathrm{~m}^{2}$. The cameras are located about $4 \mathrm{~m}$ downstream of the model, $2 \mathrm{~m}$ from the open jet's central axis (Fig. 3). The cameras are equipped with $50 \mathrm{~mm}$ Nikkor objectives with an aperture set to $f / 4$ and tilt adapters to satisfy the Scheimpflug condition. The optical magnification is equal to 0.0125 , resulting in a digital image resolution of $1.6 \mathrm{~mm} / \mathrm{px}$. For the geometrical

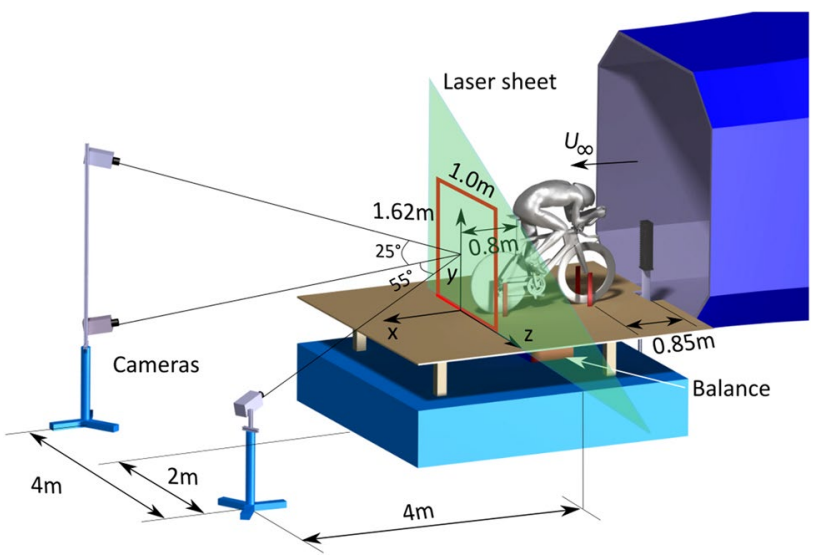

Fig. 3 Schematic representation of the experimental setup

camera calibration, an in-house developed double-plane calibration target of $1.2 \times 1.2 \mathrm{~m}$ is placed vertically at two locations, $5 \mathrm{~cm}$ upstream and $5 \mathrm{~cm}$ downstream of the measurement plane. The target contains a total of 156 circular dots of $8 \mathrm{~mm}$ diameter per plane, distributed over 12 rows and 13 columns with a pitch of $9 \mathrm{~cm}$ in both vertical and horizontal direction. The offset between the two planes is $2 \mathrm{~cm}$; the dots of the two planes are staggered by $4.5 \mathrm{~cm}$ in both the vertical and horizontal directions.

\subsection{PTV measurement procedure}

The images are recorded in short bursts of 11 images at $4 \mathrm{kHz}$ acquisition frequency resulting in a mean track length of five particles irrespective of the transverse location on the wake plane. Particle streaks, visualized as the maximum image intensity over the 11 subsequent images of a burst, in the centre of the model's wake and in the freestream are depicted in Fig. 4-left and right, respectively. The number of particles per pixel (ppp) varies between 0.04 and 0.1 depending on the seeder position. The highest density is observed in the freestream, where the seeded streamtube remains largely unaffected by the wake of the model, whereas the lowest ppp appears in the cyclist's wake as the particles spread over a larger volume. The average particle image intensity is rather independent of the seeder position and is approximately equal to 200 counts over a background intensity of about 20 counts, resulting in an image signal-to-noise ratio of 10 . For each position of the seeding generator, 480 bursts are acquired with $0.1 \mathrm{~s}$ separation between two successive bursts to obtain a statistical ensemble of uncorrelated particle tracks. Image acquisition and processing is conducted with DaVis 8.4 from LaVision GmbH. 
Fig. 4 HFSB seeding in the wake of the model. Particle streaks without pre-processing in the central wake (left) and after pre-processing by timeaverage intensity subtraction in the freestream area (right)
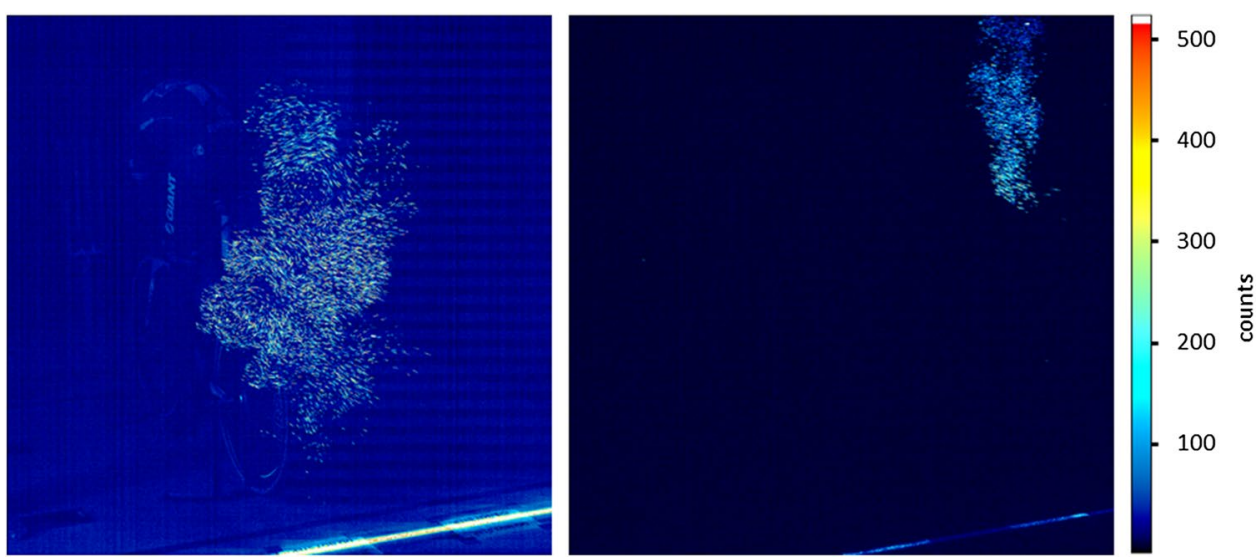

\subsection{Force balance measurements}

Force measurements are carried out with a six-component balance designed, manufactured and calibrated by the Dutch Aerospace Laboratory (NLR). Under simultaneous loading of all six components (three forces and three moments), the balance is capable of measuring loads up to $250 \mathrm{~N}$ in the streamwise direction with a maximum uncertainty of $0.06 \%$ of the full-scale value (Alons 2008). The balance is mounted directly under the ground plate, shielded from the air flow, and connected to the bike supports (see Fig. 3). The balance measurements are conducted at each position of the seeding rake simultaneously to the PIV measurements; the acquisition frequency is set to $2 \mathrm{kHz}$ and the observation time is $30 \mathrm{~s}$.

PIV and force balance measurements are repeated at five different freestream velocities between $12.5 \mathrm{~m} / \mathrm{s}$ and $15 \mathrm{~m} / \mathrm{s}$, corresponding to $\operatorname{Re}=5 \times 10^{5}$ to $\operatorname{Re}=6 \times 10^{5}$ based on the torso length. Table 1 provides an overview of the data acquisition parameters and the varying experimental conditions.

\subsection{PIV data reduction}

The acquired images are pre-processed by subtraction of the time-averaged intensity of each burst to remove background noise (see Fig. 4-left vs right for a raw and processed image, respectively) and are then processed with the Shake-The-Box algorithm (STB; Schanz et al. 2016) yielding Lagrangian particle tracks. Particle tracks resulting from one image burst per seeder location are depicted in Fig. 5 (colours indicate different seeder positions) illustrating the extent of overlap of tracks stemming from the different positions.

Velocity statistics (time-average and fluctuations rootmean-square) are computed from the Lagrangian velocity ensemble within bins of size $5 \times 4 \times 4 \mathrm{~cm}^{3}$ with $0 \%, 75 \%$ and $75 \%$ overlap in $x$-, $y$-, and $z$-direction, respectively (Agüera et al. 2016). The resulting velocity field is defined on a Cartesian grid with a vector pitch of $1 \mathrm{~cm}$ along $y$ and $z$-directions. The bin size was determined requiring a minimum number of 25 tracks per bin. A universal outlier detection filter (Westerweel and Scarano 2005) was applied to the particle velocity data in each bin, to reduce spurious tracks, resulting in an average number of used tracks per bin of approximately 2000. The uncertainty of the velocity data is estimated comparing the time-average streamwise velocity obtained from different seeder positions in a region of overlap. Discrepancies of approximately 5\% and 2\% are obtained in the wake and the freestream, respectively.

For the computation of the aerodynamic drag via Eq. 2, apart from the velocity statistics in the wake plane, the freestream velocity $U_{\infty}$ and the static pressure are needed. The measured freestream velocity, $U_{\infty \text {,meas }}$ is obtained as the mean streamwise velocity over the three free boundaries of

Table 1 Data acquisition parameters and experimental conditions

\begin{tabular}{lll}
\hline Acquisition parameters & PIV & $\begin{array}{l}\text { Bursts of } 11 \text { images at } 4 \mathrm{kHz} \\
480 \text { image bursts at } 10 \mathrm{~Hz}\end{array}$ \\
& Force balance & $\begin{array}{l}\text { Acquisition at } 2 \mathrm{kHz} \\
\text { Observation time of } 30 \mathrm{~s}\end{array}$ \\
& Freestream velocity & $U_{\infty}=[12.95,13.34,13.71,14.33,14.78] \mathrm{m} / \mathrm{s}$ \\
$\begin{array}{l}\text { Experimental conditions (for PIV and } \\
\text { balance) }\end{array}$ & Seeding system & $\begin{array}{l}15 \text { different positions; } 5 \text { along the horizontal direction and } \\
3 \text { along the vertical direction (details are provided in the } \\
\text { appendix) }\end{array}$ \\
\hline
\end{tabular}



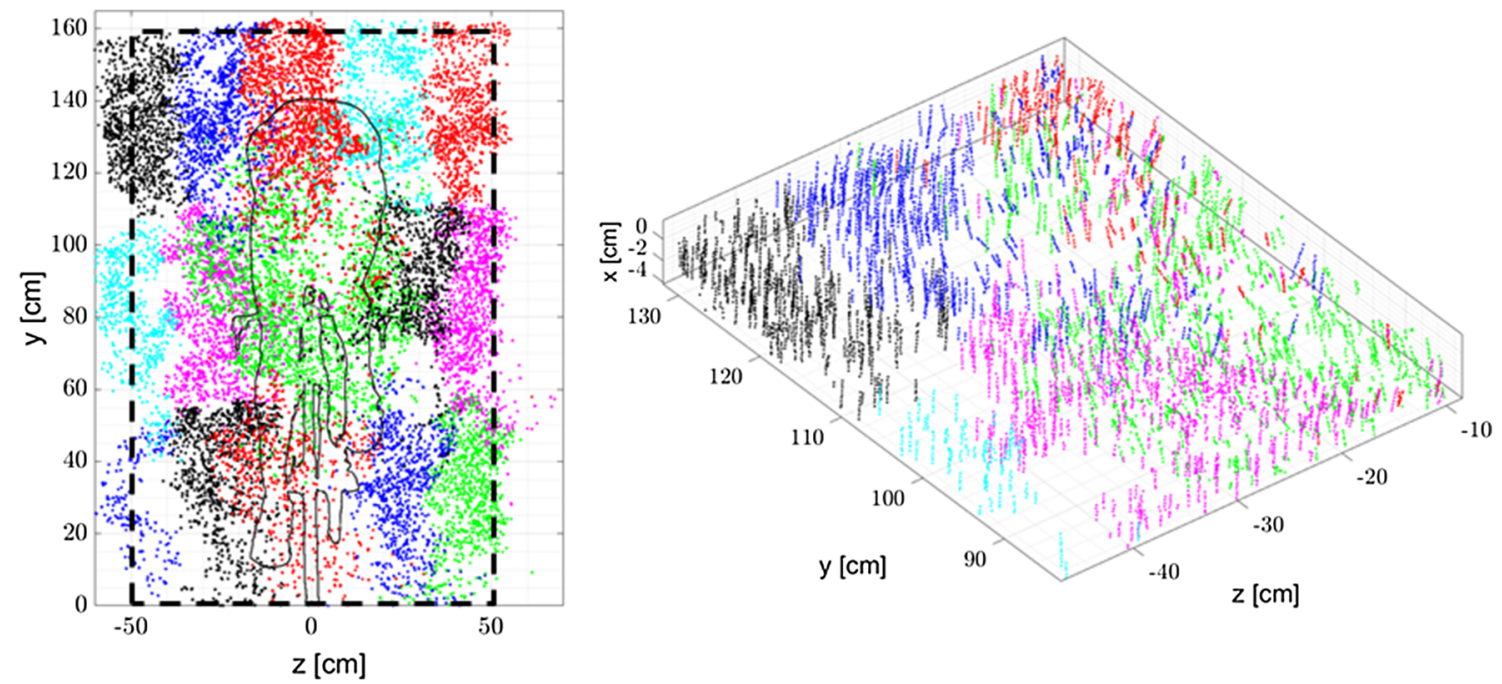

Fig. 5 Lagrangian particle tracks in the wake of the model from one image burst per seeder location (different colours represent the different seeder locations)

the wake plane, excluding a region in relative proximity to the floor $(y>50 \mathrm{~cm})$. Afterwards, a correction for the wind tunnel jet expansion, $\varepsilon_{S}$ and the nozzle blockage, $\varepsilon_{N}$ is applied according to Mercker and Wiedemann (1996), to obtain $U_{\infty}$ :

$\frac{U_{\infty}}{U_{\infty, \text { meas }}}=1+\varepsilon_{\mathrm{S}}+\varepsilon_{\mathrm{N}}$

with $\varepsilon_{\mathrm{S}}=0.0018$ and $\varepsilon_{\mathrm{N}}=0.0132$. The static pressure is obtained solving the Poisson equation for pressure prescribing Neumann conditions on the bottom boundary and Dirichlet conditions with freestream pressure on the three free boundaries. For the pressure reconstruction, the streamwise gradients of the time-average velocity and of the velocity fluctuations are neglected after estimating that these are two orders of magnitude smaller than the corresponding in-plane gradients.

Finally it should be noted that the advantage of the present PTV measurement approach, opposed to using conventional stereo-PIV, is that a stitching procedure of the velocity information obtained from the different seeder positions is not necessary. Stitching of velocity fields may result in anomalies in the velocity gradients in the overlap regions (Shah 2017), which yield reduced accuracy in the pressure field reconstruction from the solution of the Poisson equation.

\section{Results}

\subsection{Wake flow topology}

The obtained time-average flow topology in the wake of the cyclist mannequin is discussed in terms of the spatial distribution of the streamwise velocity (Fig. 6-left) and vorticity
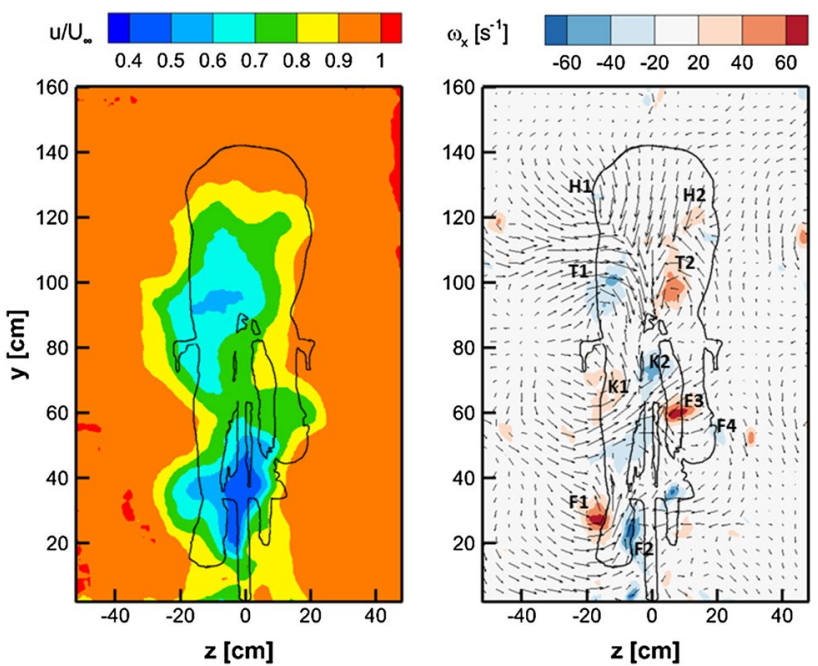

Fig. 6 Contours of time-average streamwise velocity (left) and streamwise vorticity with in-plane velocity vectors (right). Freestream velocity equal to $14.5 \mathrm{~m} / \mathrm{s}\left(R e=5.8 \times 10^{5}\right)$

with in-plane vectors (Fig. 6-right). The streamwise velocity contour exhibits two main regions of significant velocity deficit. The first one is located behind the lower back of the mannequin $(y \sim 100 \mathrm{~cm})$ slightly towards the left, and features a minimum velocity of $u / U_{\infty} \sim 0.6$. The lateral asymmetry of this velocity deficit originates from the asymmetric leg position (left leg extended downwards and right leg bent upwards), and agrees well to literature (e.g., Crouch et al. 2014; Jux et al. 2018). The minimum value of the streamwise velocity is comparatively higher than that measured by Crouch et al. (2014, $\left.u / U_{\infty} \sim 0.5\right)$ and Jux et al. $\left(2018, u / U_{\infty}\right.$ $\sim 0.35$ ). The smaller velocity deficit is attributed to the more streamlined position of the mannequin; The trunk angle of 
attack is smaller $\left(\alpha \sim 5^{\circ}\right.$ for the present model as compared to $\alpha=12.5^{\circ}$ for that used by Crouch et al.) and the head and helmet do not contribute to the frontal area of the model in contrast to the one used by Crouch et al. Although the same model was used in the case of Jux et al., the measurement plane in their case was further upstream $(x=30 \mathrm{~cm}) \mathrm{com}-$ pared to the present case $(x=80 \mathrm{~cm})$.

A second region of high velocity deficit is observed downstream of the wheel axis and the drivetrain configuration $(y \sim 40 \mathrm{~cm})$. In this region the minimum velocity of $u / U_{\infty} \sim 0.45$ matches well the work of Crouch et al. (2016) in terms of location and magnitude, despite the differences between the respective models.

A region of strong downwash behind the curved back of the mannequin $(\mathrm{y} \sim 120 \mathrm{~cm})$ is observed in Fig. 6-right, with a peak vertical velocity of $v / U_{\infty} \sim-0.17$ that agrees well with literature (Crouch et al. 2016; Griffith et al. 2014, among others). Two distinct counter-rotating vortices (marked $T 1 / T 2$ in Fig. 6-right) originate from the cyclist's thighs and are fed by the downwash behind the model's back, as also documented in previous literature (Crouch et al. 2014). Other counter-rotating vortex pairs originate from the left foot (marked F1/F2 in Fig. 6-right) and from the right foot (marked $F 3 / F 4$ ) agreeing well with the work of Jux et al. (2018). The location and strength of the streamwise vortices $K 1$ and $K 2$ also match well with the latter work. The regions of streamwise vorticity emanating from the hips $(H 1 / H 2)$ are shearing regions, rather than vortex regions, stemming from the interaction between the downwash over the back of the mannequin and the surrounding streamwise velocity. Overall, it is concluded that the measured timeaverage streamwise velocity and vorticity are in good agreement with existing work providing confidence in the quality of the present measurement approach.

The streamwise velocity fluctuations in the wake of a cyclist in time-trial position have not been reported in literature and are discussed hereafter. Two separated unsteady shear layers behind the lower left leg can be observed in Fig. 8-left (marked S3/S4), exhibiting peaks of about $\sqrt{\overline{u^{\prime 2}}} / U_{\infty} \sim 0.1$. These shear layers bend inwards just below the knee due to the strong inward velocity component in this region $(y \sim 50 ; z \sim-20$, Fig. 6-right), partly stemming from the counter-clockwise streamwise vortex $\mathrm{K} 1$. The shear layer originating from the top part of the extended leg $(y \sim 90 \mathrm{~cm}$ and $z \sim-15 \mathrm{~cm})$ exhibits fluctuations of similar magnitude. Conversely, the streamwise velocity fluctuations behind the bent leg are comparatively lower, with peaks of about $\sqrt{\overline{u^{\prime 2}}} / U_{\infty} \sim 0.06$, due to its more streamwise orientation. The location of counter-rotating streamwise vortex pairs originating from the thighs ( $T 1 / T 2$ Fig. 6-right) and the shearing regions $(H 1 / H 2)$ coincides with two regions of high streamwise
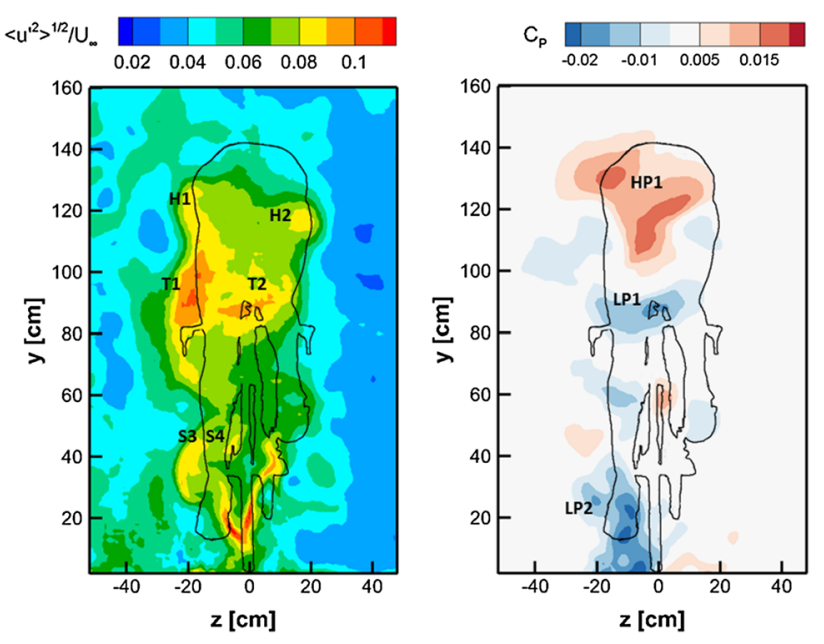

Fig. 7 Contours of streamwise velocity fluctuations (left) and time-average pressure coefficient (right) at a freestream velocity of $14.5 \mathrm{~m} / \mathrm{s}\left(\operatorname{Re}=5.8 \times 10^{5}\right)$

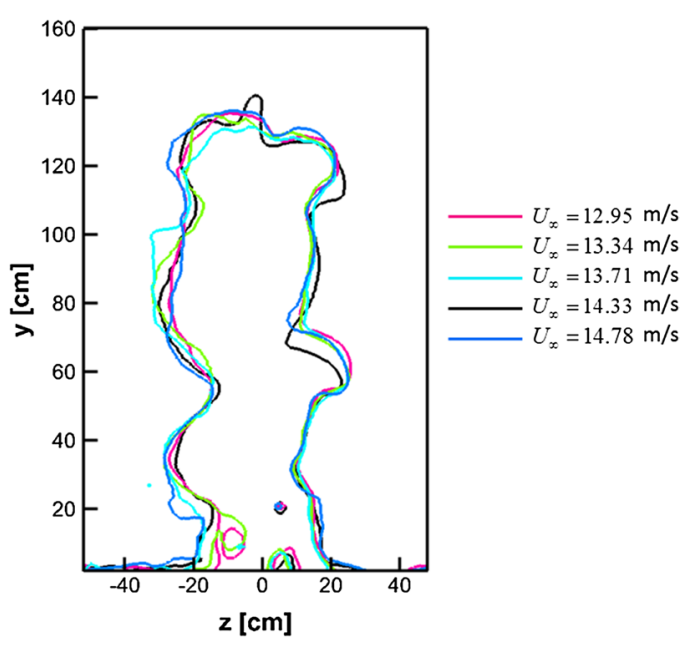

Fig. 8 Contours of $90 \% u / U_{\infty}$ at the different freestream velocities

velocity fluctuations (T1/T2 and $H 1 / H 2$ Fig. 7-left) indicating that these flow structures are unsteady in nature. Hence, the presented time-average streamwise vorticity levels in Fig. 6-right are below the instantaneous peak values suggesting that insight into the instantaneous vortex topology may contribute to a better understanding of the streamwise vortex structures in the wake of a cyclist, possibly aiding in future drag minimization. Finally, other local maxima of the streamwise velocity fluctuations appear in the wake of the drivetrain and behind the lower part of the wheel (V-shape area).

Outside of the wake of the mannequin, the root-meansquare of the streamwise velocity fluctuations reduces significantly, reaching a level of about $4 \%$ of the freestream velocity. With an estimated freestream turbulence level in 
the wake of the seeding system of $1.9 \%$ (Jux et al. 2018), it is argued that the freestream turbulence intensity is likely overestimated due to measurement random errors of the large-scale PTV system. As a consequence, the contribution of the Reynolds stress term in the expression for the drag (second term in Eq. 2) is overestimated, thus yielding an underestimation of the aerodynamic drag by approximately $0.15 \mathrm{~N}$.

At sufficient distance behind a bluff body, the contribution of the pressure term to the aerodynamic drag decays to zero due to the pressure recovery towards the freestream condition (Terra et al. 2017). To understand the contribution of the time-average pressure term to the drag for the present downstream position of the wake plane, the pressure field distribution is investigated. Figure 7-right depicts the spatial distribution of the pressure coefficient showing the presence of a large high pressure region (HP1) behind the upper back of the cyclist. This overpressure is attributed to the deceleration of the flow after passing the curved back of the cyclist. Lower in the wake plane, at $y \sim 90 \mathrm{~cm}$, the flow has separated over the lower back of the cyclist, resulting in a region of negative $C_{\mathrm{P}}$ (LP1). A second low pressure region, likely caused by a flow separation over the left foot and the rear wheel, is observed behind the left foot (LP2). The overall distribution of the time-average pressure coefficient matches well to the work of Blocken et al. (2013), despite the differences in model geometry and crank angle (symmetric leg position instead of the present asymmetric case). Finally it is observed that the spatial variations of the pressure coefficient are small (up to 0.03 between minimum and maximum $C_{\mathrm{p}}$ in the wake plane) and the pressure in most of the domain equals the freestream pressure, suggesting that the contribution of the pressure term to the aerodynamic drag (through Eq. 2) may be assumed negligible. This is discussed in more detail in the following section.

The present experiment is repeated within a narrow range of freestream velocities $\left(12.5 \mathrm{~m} / \mathrm{s}<U_{\infty}<15 \mathrm{~m} / \mathrm{s}\right)$ where the drag coefficient is constant (Grappe 2009) and, hence, the flow topology is expected to remain unaltered. The contours of $90 \%$ of $u / U_{\infty}$ at five freestream speeds, depicted in Fig. 8, coincide well and discrepancies of about 5\% in nondimensional streamwise velocity are observed between the different freestream conditions, indicating a good level of repeatability of the experiment.

\subsection{Drag estimation}

Considering the unaltered flow topology at the different freestream velocities (Fig. 8), the drag coefficient of the cyclist can be assumed constant and the aerodynamic drag is expected to scale quadratically with increasing velocity. Hence, despite the narrow range of freestream velocities, the drag force is expected to increase by almost $50 \%$. This expected increase is clearly observed in Fig. 9 depicting the resistive force obtained by the balance system (black symbols) and from the PIV wake rake (red symbols) at five freestream velocities. The uncertainty of the timeaverage balance readings is below $0.2 \mathrm{~N}$ and, hence, error bars depicting their uncertainty are omitted. A quadratic fit through the five data points resulting from the PIV wake rake $\left(D=0.1433 U_{\infty}{ }^{2}\right.$; red-dashed line $)$ and $(0,0)$ is included as well. The accuracy of the obtained drag from the PIV wake rake is estimated from the root-mean-square of the residuals between the measured data and its quadratic fit and is equal to $\varepsilon_{\mathrm{RMS}}=1.2 \mathrm{~N}$.

The accuracy of the drag estimation, or drag resolution, is also evaluated comparing the drag coefficients obtained with the PIV wake rake with those obtained by force balance. Figure 9-left shows that the drag coefficients measured by the force balance are relatively constant (black line) and the variations are obtained within $1.5 \%$ with increasing freestream velocity. The error bars on the time-average drag coefficient indicate the uncertainty of the mean value at one sigma level. Details on the computation of this uncertainty stemming from the different seeder positions are provided in the "Appendix". In contrast to the measurements by the force balance, the variations observed in the drag coefficient obtained from the PIV wake rake are significantly larger (Fig. 10-left red line), illustrating the higher measurement uncertainty of this technique. Figure 10-right (solid-red line) shows the error in the drag coefficient measured by the PIV wake rake approach relative to that obtained by balance measurements, which varies between 0.75 and $6.5 \%$. Using Eq. 3, a drag resolution of the PIV wake rake of $\Delta C_{D}=$ 0.03 or 30 drag counts, is obtained (Fig. 10-right dashedred line).

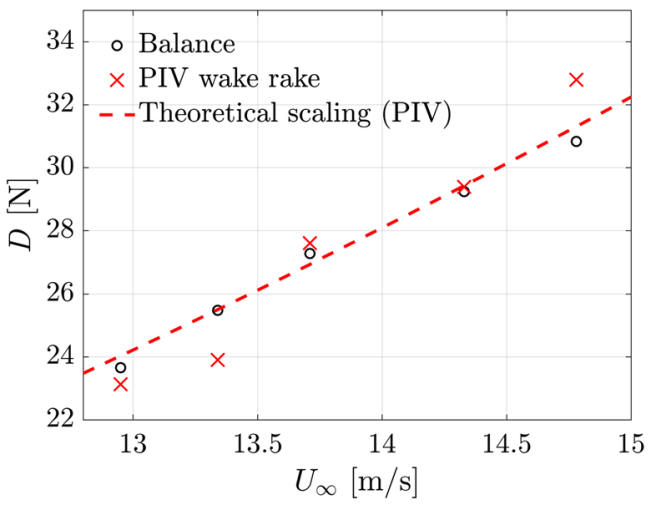

Fig. 9 Time-averaged aerodynamic drag from the balance (black symbols) and the PIV wake rake (red symbols) at five freestream velocities. The red-dashed line depicts a quadratic fit through the lat$\operatorname{ter}\left(D=0.143 U_{\infty}{ }^{2}\right)$ 
Fig. 10 Aerodynamic drag from the PIV wake rake vs force balance (left) and the relative error of the drag of the PIV wake rake as a percentage of the balance data (right)
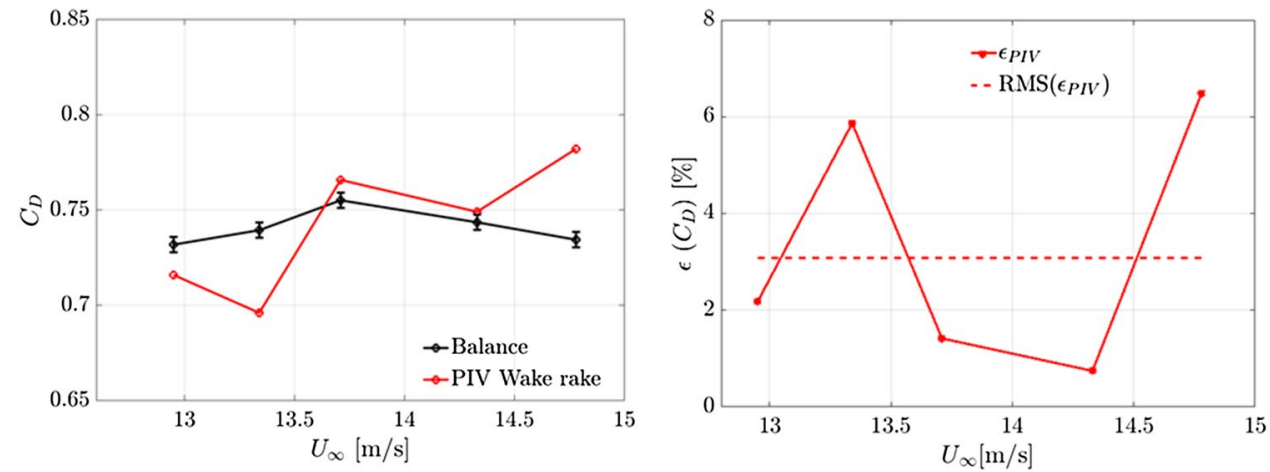

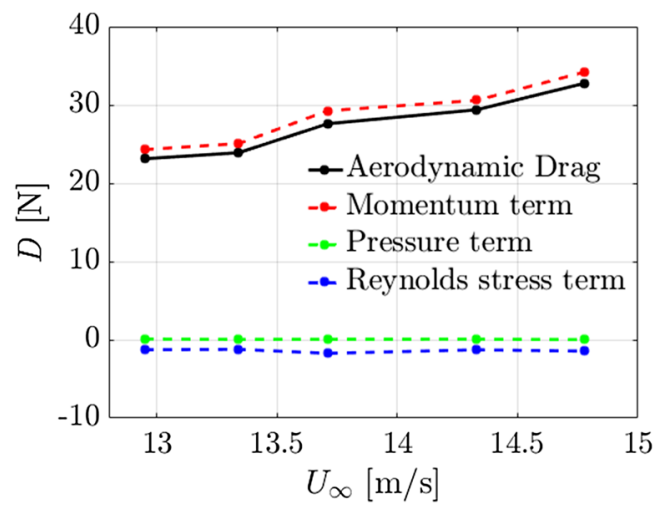

Fig. 11 Time-average aerodynamic drag from the PIV wake rake at five freestream velocities including the individual momentum, pressure and Reynolds stress term

Finally, the separate contributions of the momentum term, Reynolds stress term and pressure term (Eq. 2) to the overall aerodynamic drag are depicted in Fig. 11. The contribution of the latter is approximately zero at all freestream conditions, which was expected from the small zero-level deviations in the distribution of the pressure coefficient (Fig. 7-right). Hence, the pressure reconstruction can be omitted in future cyclist drag estimations with a wake plane downstream position $x / L>2.2$, where $L$ is the characteristic length scale representative for the wake topology (hip width in this case), significantly simplifying the evaluation of the aerodynamic drag. The Reynolds stress term consistently contributes by approximately $5 \%$ of the drag with increasing freestream velocity and cannot be neglected. Finally, the momentum term dominates the air resistance accounting for the remaining $95 \%$.

\section{Conclusions}

Large-scale PTV measurements have been conducted in thin volume of $5 \times 100 \times 160 \mathrm{~cm}^{3}$ in the wake of a full-scale cyclist model. Cameras and laser imaged and illuminated, respectively, the entire measurement domain, while an in-house built HFSB seeding generator was traversed into 15 different positions to obtain flow tracers in the whole domain. The distribution of time-average streamwise velocity, vorticity and pressure coefficient resembles literature closely and a good level of repeatability is demonstrated iterating the measurements in a narrow range of Reynolds numbers $\left(5 \times 10^{5}<R e<6 \times 10^{5}\right)$. The main streamwise vortices in the wake of the mannequin are clearly unsteady in nature, suggesting that an analysis of the instantaneous wake topology may provide new insights for future drag reductions. By invoking the conservation of momentum in a control volume containing the model, the time-average aerodynamic drag acting on the wind tunnel model is expressed as a summation of three terms composing the overall resistive force, namely, the momentum, the Reynolds stress and the pressure term. In the present conditions, the contribution of the pressure is negligible and, hence, the pressure reconstruction can be omitted for drag evaluation at a downstream distance of $x / L \geq 2.2$, where $L$ is the hip width. Conversely, the momentum term dominates the overall drag force, contributing to about $95 \%$ of the drag value. The drag resolution of the PIV wake rake technique is estimated comparing the aerodynamic drag to that of a force balance at five freestream velocities, resulting in a measurement sensitivity of 30 drag counts or $\Delta C_{D}$ $=0.03$. Although this qualifies the large-scale PIV wake rake as a rather coarse instrument for drag determination, increased accuracy can be expected using a larger seeding system to avoid traversing the seeder, and placing it inside the settling chamber of the wind tunnel to decrease the induced flow disturbance.

Open Access This article is distributed under the terms of the Creative Commons Attribution 4.0 International License (http://creativeco mmons.org/licenses/by/4.0/), which permits unrestricted use, distribution, and reproduction in any medium, provided you give appropriate credit to the original author(s) and the source, provide a link to the Creative Commons license, and indicate if changes were made. 
Fig. 12 Seeder position numbering and illustration of seeder position 2 (left) and the effect of the seeder on the measured aerodynamic drag with the balance at $U_{\infty}=12.97 \mathrm{~m} / \mathrm{s}$ (right)

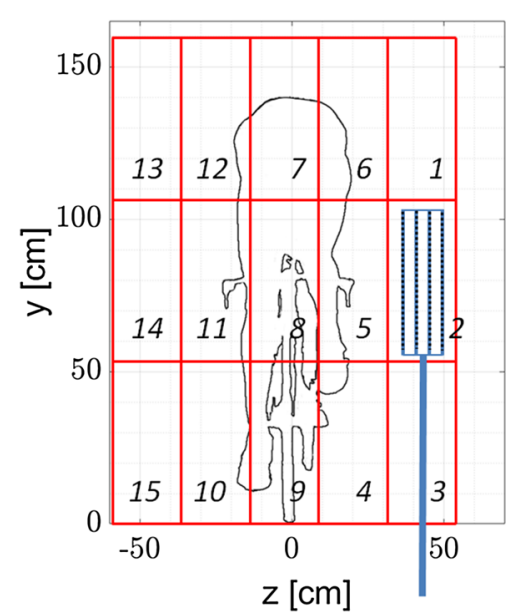

\section{Appendix}

Balance measurements are conducted simultaneously to PTV at each position of the seeding system. The HFSB seeder is traversed into 15 positions, depicted in Fig. 12left. For each position the obtained drag force is presented in Fig. 12-right. Clearly, the measured drag at positions 7 and 8 is largely affected by the seeder upstream of the model, with a drag value 4 to $8 \%$ below that of the other positions. In these cases, the lateral position of the supporting strut of the seeder coincides with that of the wind tunnel model and, hence, the velocity deficit in the wake of the former reduces the drag measured on the latter. This reduction in drag, however, is not observed for seeder position 9. In this position the strut supporting the seeder is entirely below the ground plate and its wake cannot interact with the wind tunnel model. Therefore, it is concluded that it is mainly the wake of this strut, and not that of the seeder itself, that interacts with the bike and the mannequin and reduces its drag. In the wake of the strut, generally, no or little seeding is present and, hence, no PTV measurements are conducted. Hence, it is assumed that the wake of the strut has a negligible effect on the measured velocity and the resulting drag measured by the PIV wake rake. To compare the drag obtained by the PIV wake rake and that of the force balance, therefore, the measurements of the latter that are affected by the strut are excluded. To identify these data points, the drag values outside one standard deviation from the mean obtained over all seeder positions are excluded in the computation of the final statistical mean aerodynamic drag. The standard deviation of the data excluding these outliers is considered the uncertainty of the mean drag and is presented by the error bars in Fig. 10.

\section{References}

Agüera N, Cafiero G, Astarita T, Discetti S (2016) Ensemble 3D PTV for high resolution turbulent statistics. Meas Sci Technol 27:124011

Alons HJ (2008) OJF External Balance. Nationaal Lucht en Ruimtevaartlaboritorium (National Aerospace Laboratory NLR), NLR-CR-2008-695

Anderson JD Jr (1991) Fundamentals of aerodynamics. International edn. McGraw-Hill, New York

Barry N, Burton D, Sheridan J, Thompson M, Brown NAT (2014) Aerodynamic performance and riding posture in road cycling and triathlon. Proc IMechE Part P J Sports Eng Tech 229:1-11

Betz A (1925) A method for the direct determination of profile drag (in German). Zeitschrift für Flugtechnik Motorluftschifffahrt 16:42-44

Blocken B, Defraeye T, Koninkx E, Carmeliet J, Hespel P (2013) CFD simulations of the aerodynamic drag of two drafting cyclists. Comput Fluids 71:435-445

Bosbach J, Kühn M, Wagner C (2009) Large scale particle image velocimetry with helium filled soap bubbles. Exp Fluids 46:539_ 547. https://doi.org/10.1007/s00348-008-0579-0

Caridi GCA, Ragni D, Sciacchitano S, Scarano F (2016) HFSB-seeding for large-scale tomographic PIV in wind tunnels. Exp Fluids 57:190. https://doi.org/10.1007/s00348-016-2277-7

Crouch TN, Burton D, Brown NAT, Thomson MC, Sheridan J (2014) Flow topology in the wake of a cyclist and its effect on aerodynamic drag. J Fluid Mech 748:5-35. https://doi.org/10.1017/ jfm.2013.678

Crouch TN, Burton D, Thompson MC, Brown NAT, Sheridan J (2016) Dynamic leg-motion and its effect on the aerodynamic performance of cyclists. J Fl Str 65:121-137

De Kat R, van Oudheusden BW (2012) Instantaneous planar pressure determination from PIV in turbulent flow. Exp Fluids 52:1089_ 1106. https://doi.org/10.1007/s00348-011-1237-5

Defraeye T, Blocken B, Koninckx E, Hespel P, Carmeliet J (2010) Computational fluid dynamics analysis of cyclist aerodynamics: performance of different turbulence-modelling and boundarylayer modelling approaches. J Biomech 43:2281-2287

Goett HJ (1938) Experimental investigation of the momentum method for determining profile drag. NACA Annu Rep 25:365-371

Grappe F (2009) Résistance totale qui s'oppose au déplacement en cyclisme [Total resistive forces opposed to the motion in cycling]. In: Cyclisme et Optimisation de la Performance [Cycling and 
optimisation of performance], 2nd edn. De Boeck Université, Collection Science et Pratique du Sport, Paris, p 640

Griffith MD, Crouch T, Thompson MC, Burton D, Sheridan J, Brown NA (2014) Computational fluid dynamics study of the effect of leg position on cyclist aerodynamic drag. J Fluids Eng 136(10): 101105

Hucho W, Sovran G (1993) Aerodynamics of road vehicles. Annu Rev Fluid Mech 25:485-537. https://doi.org/10.1146/annur ev.fl.25.010193.002413

Jones BM (1936) Measurement of profile drag by the pitot-traverse method. ARC R\&M No. 1688

Jux C, Sciacchitano A, Schneiders JFG, Scarano F (2018) Robotic volumetric PIV of a full-scale cyclist. Exp Fluids 59:74. https:// doi.org/10.1007/s00348-018-2524-1

Kurtulus DF, Scarano F, David L (2007) Unsteady aerodynamic forces estimation on a square cylinder by TR-PIV. Exp. Fluids 42:185-196

Lentink D (2018) Accurate fluid force measurement based on control surface integration. Exp Fluids 59:22. https://doi.org/10.1007/ s00348-017-2464-1

Lignarolo LEM, Ragni D, Krishnaswami C, Chen Q, Simão Ferreira CJ, Van Bussel GJW (2014) Experimental analysis of the wake of a horizontal-axis wind-turbine model. Renew Energy 70:31-46

Lin JC, Rockwell D (1996) Force identification by vorticity fields: techniques based on flow imaging. J Fluids Struct 10:663-668

Maskell EC (1973) Progress towards a method for the measurement of the components of the drag of a wing of finite span. RAE technical report 72232

Mercker E, Wiedemann J (1996) On the correction of interference effects in open jet wind tunnels. SAE Trans J Eng 105(6):795-809

Noca F, Shiels D, Jeon D (1997) Measuring instantaneous fluid dynamic forces on bodies, using only velocity fields and their derivatives. J Fluids Struct 11:345-350

Raffel M, Willert CE, Scarano F, Kähler CJ, Wereley ST, Kompenhans J (2018) Particle image velocimetry—a practical guide. Springer International Publishing, Cham

Ragni D, Ashok A, van Oudheusden BW, Scarano F (2009) Surface pressure and aerodynamic loads determination of a transonic airfoil based on particle image velocimetry. Meas Sci Technol 20:074005

Ragni D, van Oudheusden BW, Scarano F (2011) Non-intrusive aerodynamic loads analysis of an aircraft propeller blade. Exp Fluids 51:361-371

Rival DE, van Oudheusden BW (2017) Load-estimation techniques for unsteady incompressible flows. Exp Fluids 58:20. https://doi. org/10.1007/s00348-017-2304-3
Scarano F, Ghaemi S, Caridi GCA, Bosbach J, Dierksheide U, Sciacchitano A (2015) On the use of helium-filled soap bubbles for large-scale tomographic PIV in wind tunnel experiments. Exp Fluids 56:42. https://doi.org/10.1007/s00348-015-1909-7

Schanz D, Gesemann S, Schroeder A (2016) Shake The Box: Lagrangian particle tracking at high particle image densities. Exp Fluids $57-70$

Schneiders JFG, Caridi GCA, Sciacchitano A, Scarano F (2016) Large-scale volumetric pressure from tomographic PTV with HFSB tracers. Exp Fluids 57:164. https://doi.org/10.1007/s0034 8-016-2258-x

Shah YH (2017) Drag analysis of full scale cyclist model using largescale 4D-PTV. MSc Thesis, Delft University of Technology, Department of Aerospace Engineering

Somers DM (1997) Design and experimental results for the S809Airfoil. NREL1SR-440-6918

Terra W, Sciacchitano A, Scarano F (2016) Evaluation of aerodynamic drag of a full-scale cyclist model by large-scale tomographic PIV. In: International workshop on non-intrusive optical flow diagnostics, Delft, 25-26 Oct 2016

Terra W, Sciacchitano A, Scarano F (2017) Aerodynamic drag of a transiting sphere by large-scale tomographic PIV. Exp Fluids 58:83. https://doi.org/10.1007/s00348-017-2331-0

Tropea C, Yarin A, Foss JF (2007) Springer handbook of experimental fluid mechanics. Springer, Berlin (ISBN 978-3-540-30299-5)

Unal MF, Lin JC, Rockwell D (1997) Force prediction by PIV imaging: a momentum based approach. J Fluids Struct 11:965-971

Van Oudheusden BW (2013) PIV-based pressure measurement. Meas Sci Technol 24:032001. https://doi.org/10.1088/09570233/24/3/032001

Van Oudheusden BW, Scarano F, Casimiri EWF (2006) Non-intrusive load characterization of an airfoil using PIV. Exp Fluids 40:988992. https://doi.org/10.1007/s00348-006-0149-2

Van Tubergen J, Verlinden J, Stroober M, Baldewsing R (2017) Suited for performance: fast full-scale replication of athlete with FDM. In: Proceedings of SCF'17. Cambridge, MA, USA, June 12-13 2017

Westerweel J, Scarano F (2005) Universal outlier detection for PIV data. Exp in Fluids 39(6):1096-1100. https://doi.org/10.1007/ s00348-005-0016-6

Zdravkovich MM (1992) Aerodynamic of bicycle wheel and frame. J Wind Eng Ind Aerodyn 40:55-70. https://doi.org/10.1016/01676105(92)90520-K

Publisher's Note Springer Nature remains neutral with regard to jurisdictional claims in published maps and institutional affiliations. 